\title{
5G Radiation and Potential Risks to the Environment and Human Health
}

\author{
Hasan J. Hasan', Ali J. Yosef ${ }^{2}$ and Haider Abed Hachem ${ }^{3}$ \\ ${ }^{1}$ Ministry of communication-Thi_Qar communication Directorate - Iraq \\ ${ }^{2}$ Mustanseria University- Baghdad - Iraq \\ ${ }^{3}$ Ministry of Education- Dhi Qar Education Directorate \\ Email:mktbshbkh@gmail.com_alijabiryousif@gmail.com
}

Article History: Received: 10 November 2020; Revised 12 January 2021 Accepted: 27 January 2021; Published online: 5 April 2021

\begin{abstract}
:-
The rapid development in wireless communication technology and the urgent need to develop it has led to an increase in the public exposure to more radio spectrum frequencies. The mountain increases in terms of the damage caused by these wireless technologies for the $2 \mathrm{G}, 3 \mathrm{G}$ and $4 \mathrm{G}$ generations ending with the new technique, which is $5 \mathrm{G}$. As a result of the urgent need for fast communications and wide packages of data, only a new generation of communications has emerged, which is $5 \mathrm{G}$, which enjoys high speed and broadcasts in short waves and high frequency. There is great concern about the impact of these technologies on human health and safety. This technique works at relatively higher frequencies than the previously used technologies. This research sheds light on some of the expected harms of these technologies and the result of using these techniques, where the damages on the human body in general, the skin and the human eye in particular, are addressed, as well as their impact on the environment and plants in general.
\end{abstract}

Keywords - Issues of Publics Health; Environmental Issue; 5G communication, Wireless communication

\section{Introduction: -}

Use of the cellular mobiles technology remainders worldwide Rise. A new 5th generation, faster (5G) Recently, the telecommunications infrastructure has been endorsed by Federal Communications Commission (FCC) with initial antennas installation and testing are now under way. Although it may offer Uber automation and "immersive" instantaneous" us" There are a lot of concerns about entertainment with respect to public health and wireless system protection[1]. 5G The higher frequencies in millimetre waves would include the communications and internet, never before used in technology[1]. The 5G rollout recommends the addition of microwave spectrum frequencies in the medium-Mid- $(3.7 \mathrm{GHz}-24 \mathrm{GHz})$, mid- $(0.6 \mathrm{GHz}-3.7 \mathrm{GHz})$, and Highband frequencies $(24 \mathrm{GHz}$ and above for higher speeds telecommunications. Since these higher frequencies do not pass fast enough, [1].

This scheme, closed by walls, A dense network of outdoor fixed antennas every $300 \mathrm{~m}$ should be used as well as indoor devices[2]. This spectrum or radiation, like that radiation $2 \mathrm{G}, 3 \mathrm{G}$ and $4 \mathrm{G}$, telecommunication networks do not have long-term health consequences have been pre-market checked for despite the fact that it would reveal people. Fig 1. displays the MM Wave cell by low-frequency cell Frequency for wireless networking [3]. 


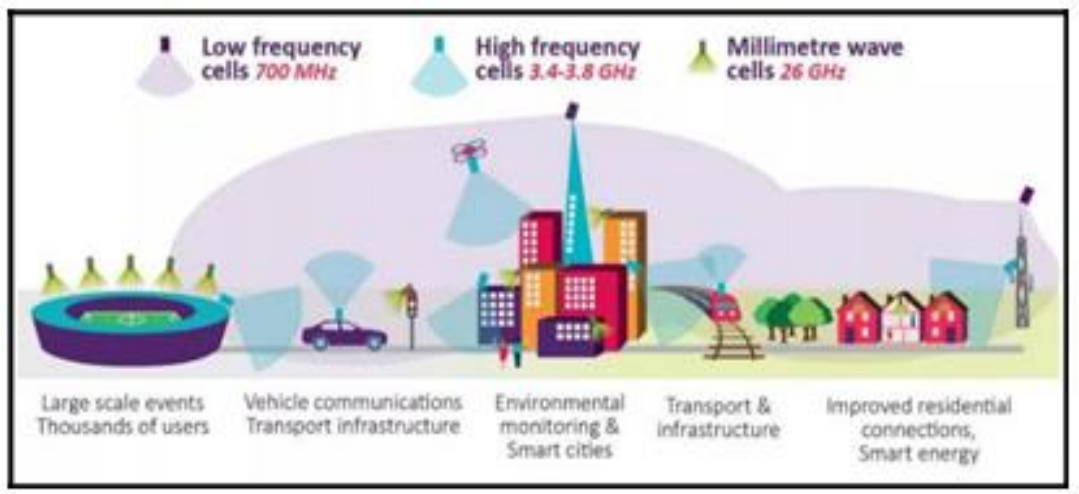

Fig. 1. Wireless communication in life

\section{Problem on Public Health}

The primary absorption of millimetre waves (MMWs) is within one to two millimetres of human skin and within one to two millimetres of humanoid skin corneal of layers' surface. Therefore, skin the main goals of the tissue zone with radiations. Then, the skin comprises Small blood vessels and nerves, bio-effects of the MMW can be transmitted via endings, by skin's molecular pathways or finished Via the system of the nervous.[4].

5G high spectrum range can lead to cell mutations It causes tumours that may become cancer later.5G radiation exposure increases the output of from ROS. Standard of Reactive Oxygen Species (ROS) are A portion of cellular processes and signalling for cells. The overproduction of non-balanced ROS With Superoxide dismutase (both endogenous antioxidants) (SOD), glutathione peroxidase (GPx), melatonin, (Vitamin C, Carotenoids, Vitamin E, Polyphenols) allow free radicals to form Oxidizing and destroying DNA, proteins and membranes Mitochondria and lipids. There are no mitochondrial histones as a consequence of that it does not restore damage to DNA and is not protected from reactive oxygen species in mitochondria.

\subsection{Skin Effects}

A variety of studies have shown the surface low-intensity MMW effects can be very important.

substantial, triggering a variety of biological modifications, And at non-thermal, like cell membrane, levels repercussions[5]. Application of those frequencies in the short term stimulates the release in the area of endogenous opioids about skin. Me be the range of MMW is appears in $95 \mathrm{GHz}$ affects and act as a dangerous material stimulus without thermal disruption or heating. The sweat glands were suggested by researchers as a Objective. Application of certain frequencies in the short term Triggers the release in the region of endogenous opioids About skin. It appears that the MMW range of $95 \mathrm{GHz}$ affects The cutaneous nociceptors which serve as a hazardous material Stimulation without thermal disturbance or heating. The sweat glands were proposed as a target by researchers. Feldman et al $(2008 ; 2009)$ found that helically shaped sweat ducts are tubes in human skin filled with an aqueous conductive solution[6].

\subsection{Effects on The eyes}

The leading cause of blindness has been cataracts. As a 5G device, there is a clear concern for $5 \mathrm{G}$ application. The eyes will also produce significant radiation from the. Well-established risk factors in cataract formation are age, exposure to smoking, diabetes, and UVB[7]. 


\subsection{Effect of Neurology}

The nervous system or the cortex when upset, EMR, Electrophysiological, Morphological, and There may be chemical shifts. A significant alteration in these positions would eventually lead to a shift in conduct. The physiological effects of EMR, yes, blood brain changes documented in the literature include barrier, electrophysiology, Anatomy, Cellular metabolism, neurotransmitter activities, Calcium efflux, drug reactions that affect the Nervous System[8].

\subsection{ISSUES OF THE ENVIRONMENTAL}

In places, Birds and other species saturated with "non-ionizing" 4G radiation are vanishing. The dreadful manmade frequencies that emanate from our machines destroy innocent lives, literally, beings or allowing them to escape en masse[9]. Humanity next is it. For any amount of time, there would be no life form that can tolerate 5G. Its appearance on our world is a genocidal, murderous attack on humanity.

\subsection{Effect on vegetation}

A research on aspen seedlings in 2010 determined Radio frequency sensitivity It has allowed leaves to show

signs of necrosis, though trials focused on Armenian Low intensity millimetre waves have been shown to evoke

isoenzyme peroxidase (a stress protein in plants) Changing the spectrum of wheat leaves. The current tense, Job, the effect of EMI EHF on total peroxidase Spectrum of activity and isoenzyme in wheat seedlings Cell have been observed[10].

\subsection{Wildlife Consequences}

With the arrival of 3G technology, there has been Increased accounts of the abandonment of their nests by birds as

As well as health concerns including the loss of plumage, Problems with locomotion, diminished survivorship[11], and death," says Alfonso Balmori, a researcher. Species of Birds Non-ionizing, which is impaired by these low levels, radiation from the MM Wave is House Sparrows, Rock Doves, Storks of Ivory, Collared Doves, and Magpies,

Among others, But it's not the birds alone. The decreasing population of bees is also said to be connected to

Radiation from this non-ionizing EMF. This decreases the egglaying process. The queen's talents have contributed to a fall in Power of the colony. In the Netherlands, an incident occurred because of In November, the 5G experiment killed 287 birds. In 2018[12].

\section{Thermal Effects Overall}

1- When receiving EM radiation, it is transformed to Into the sun. A process readily comprehensible Tissue heating is the effect of radiation. (effect of thermal). Altering their biological processes Functions as a consequence of a temperature change. When nuclear radiation takes place[13], this causes the charged particles to cause the matter to Oscillate and obtain strength. The true destiny of This energy is dependent on the scenario. It should have been It is re-radiated instantly and appears dispersed, Radiation reflected, or 
distributed. Also, it can get Dissipated into other inside microscopic movements Coming to thermal equilibrium, and the matter as thermal energy expresses itself in the Substance.

\section{2- Powerful radio waves can thermally burn living environments.}

Tissues and food can be fried. Besides infrared, Visible lasers, sufficiently powerful and ultraviolet light can set paper afire rapidly, too. Ionization High-speed electromagnetic radiation can create But several times, after these electrons collide with Eventually, much of the energy is downgraded to thermal energy by other atoms in the material[14]. This whole process occurs in a tiny fraction of the a second, in a substance, electrons and break chemical bonds.

\section{The phase of inverse or time-reversed}

Thermal radiation is responsible for absorption. The radiation that results can subsequently be Another piece of matter consumed, with The energy deposited heats the material[15]. Radiation of a significant mechanism for the transfer of heat.

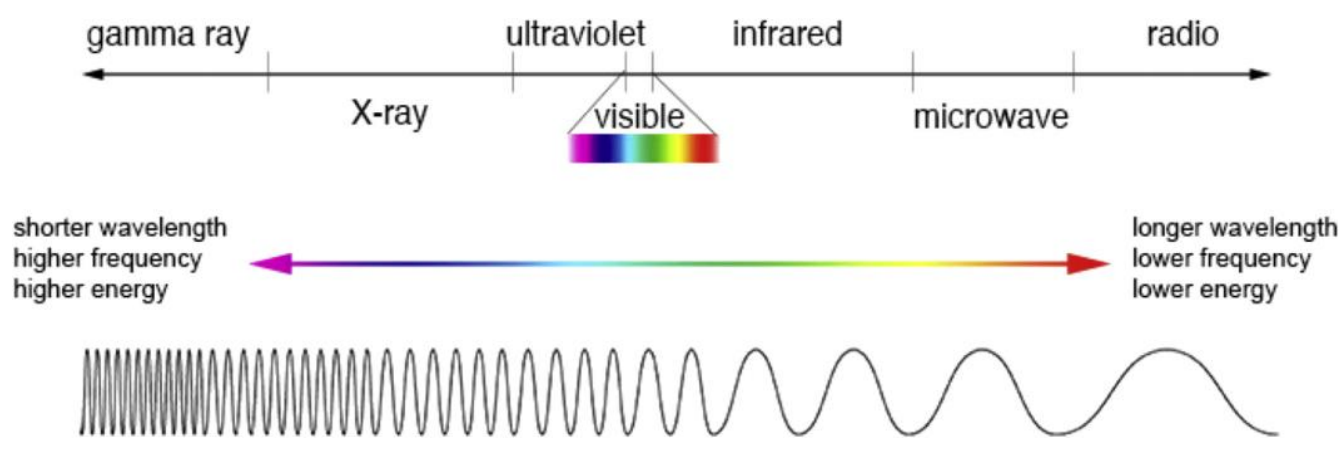

Fig 4.1The Spectrum Range[16]

\subsection{The Specific of Absorption Rate or (SAR)}

The pace at which humans consume radiation specific absorption rate (SAR), measured by the body, Measured in watts per $\mathrm{kg}(\mathrm{W} / \mathrm{kg})$ of tissue in units[17]. If The created heat is tiny, the body's Without a thermoregulatory system, it can dissipate Causing detrimental results. If it reaches the temperature This skill, approximately one to two degrees Celsius. Tissue there may be damage. Every mobile phone is shipped with A SAR ranking (although some producers are attempting to its hide). Regulatory agencies have set their maximum standard. Depending on its nature, radiation, Antenna, how to keep it and how to use it. Institute of the Engineers of Electrical and Electronics (IEEE)[18]. Guy and Radiation Committee, both national and national, Security has been developed by international organizations Guidelines for RF Energy Exposure.

\subsection{FCC Limits of 5G MMWave exposure}

For mobile phones, laptops, and others, SAR levels are used to assess regulatory enforcement, handheld wireless devices Observance. For applications with millimeter wavelengths and Power density for utilities above $6 \mathrm{GHz}$ (FCC) and There is a need to test above $10 \mathrm{GHz}$ (ICNIRP) with Density of power (FCC, 1997; Wu et al., 2015a)[5]. The greater absorption of energy in a shallow field is due to That causes more rapid heating, resulting in a lot of higher levels of SAR. Maximum Allowable Exposure (MPE) of the FCC in Power Density Conditions for frequencies between 1.5 And $100 \mathrm{GHz}$ over a duration of 30 minutes is $10 \mathrm{~mW} / \mathrm{cm} 2$ 
(FCC, 1997; 2014: Romanenko et al.). Generated Heat in handheld devices for 5G, it is a problem but still is considered the only true damage measure, no It takes into account biological cellular alterations (Wu et et The. al., 2015a).

\section{CONCLUSION}

In this period of new communication technology, there are more disadvantages than benefits, and they have often become life-threatening. 5G in MM Wave software offers high data rates and Make all related to the IOT, which can be Considered an optimistic but dangerous point Impact on human health and ecosystems of $5 \mathrm{G} \mathrm{MM}$ Wave If we try to minimize the results by attempting to reduce them, it is not zero. Given the importance of SAR, 5G software will It should be regarded as fruitful.

\section{REFRENCES: -}

[1] G. A. Akpakwu, B. J. Silva, G. P. Hancke, and A. M. Abu-Mahfouz, "A survey on 5G networks for the Internet of Things: Communication technologies and challenges," IEEE access, vol. 6, pp. 3619-3647, 2017.

[2] W. Manan, "Propagation channel models for 5G mobile networks. Simulation and measurements of 5G propagation channel models for indoor and outdoor environments covering both LOS and NLOS Scenarios," University of Bradford, 2018.

[3] B.-C. E. Ndinojuo, "5G, Religion, and Misconceptions in Communication during Covid-19 in Nigeria," Jurnal The Messenger, vol. 12, pp. 97-110, 2020.

[4] J. Moskowitz, "5G wireless technology: millimeter wave health effects," Electromagnetic Radiation Safety, vol. 3, pp. 3-6, 2017.

[5] C. L. Russell, "5 G wireless telecommunications expansion: Public health and environmental implications," Environmental research, vol. 165, pp. 484-495, 2018.

[6] Y. Feldman, A. Puzenko, P. B. Ishai, A. Caduff, I. Davidovich, F. Sakran, et al., "The electromagnetic response of human skin in the millimetre and submillimetre wave range," Physics in Medicine \& Biology, vol. 54, p. 3341, 2009.

[7] M. F. McLaughlin, D. B. Donoviel, and J. A. Jones, "Novel indications for commonly used medications as radiation protectants in spaceflight," Aerospace medicine and human performance, vol. 88, pp. 665-676, 2017.

[8] S. Goldberg, "Michigan House Energy Policy Committee 10/4/18 Testimony Key Points/References on 5G Associated Health Issues," Bioelectromagnetics, vol. 39, pp. 277-288, 2018.

[9] O. Nizhelska, L. Marynchenko, and V. Piasetskyi, "Biological Risks of Using Non-Thermal Non-Ionizing Electromagnetic Fields," 2020.

[10] K. Haggerty, "Adverse influence of radio frequency background on trembling aspen seedlings: Preliminary observations," International Journal of Forestry Research, vol. 2010, 2010.

[11] S. Sivani and D. Sudarsanam, "Impacts of radio-frequency electromagnetic field (RF-EMF) from cell phone towers and wireless devices on biosystem and ecosystem-a review," Biology and Medicine, vol. 4, p. 202, 2012.

[12] A. E. Crawley, Revival: Studies of Savages and Sex (1929): Routledge, 2018.

[13] D. Kondepudi and I. Prigogine, Modern thermodynamics: from heat engines to dissipative structures: John Wiley \& Sons, 2014.

[14] B. A. Meyers, Pemf-the Fifth Element of Health: Learn Why Pulsed Electromagnetic Field (Pemf) Therapy Supercharges Your Health Like Nothing Else!: BalboaPress, 2013.

[15] Y. V. Bykov, K. I. Rybakov, and V. Semenov, "High-temperature microwave processing of materials," Journal of Physics D: Applied Physics, vol. 34, p. R55, 2001.

[16] M. M. Ohayon, V. Stolc, F. T. Freund, C. Milesi, and S. S. Sullivan, "The potential for impact of man-made super low and extremely low frequency electromagnetic fields on sleep," Sleep medicine reviews, vol. 47, pp. 28-38, 2019.

[17] S. ul Islam, G. Ahmed, M. Shahid, N. Hassan, M. Riaz, H. Jan, et al., "Implanted Wireless Body Area Networks: Energy Management, Specific Absorption Rate and Safety Aspects," in Ambient Assisted Living and Enhanced Living Environments, ed: Elsevier, 2017, pp. 17-36.

[18] K. Jomaa, "Electromagnetic Near-field Characterization \& Occupational Exposure to RF Waves in Industrial Environment," 2018. 